\title{
A Unified Framework to Quantify the Credibility of Scientific Findings
}

\author{
Etienne P. LeBel \\ University of Western Ontario \\ Institute for Globally Distributed Open Research and Education (IGDORE) \\ Randy McCarthy \\ Northern Illinois University \\ Brian D. Earp \\ University of Oxford \\ Malte Elson \\ Ruhr University Bochum \\ Wolf Vanpaemel \\ University of Leuven - KU Leuven
}

\begin{abstract}
Societies invest in scientific studies to better understand the world, and attempt to harness such improved understanding to address pressing societal problems. Published research, however, can only be useful for theory or application if it is credible. In science, a credible finding is one that has repeatedly survived risky falsification attempts. However, state-of-theart meta-analytic approaches cannot determine the credibility of an effect because they do not account for the extent to which each included study has survived such attempted falsification. To overcome this problem, the following paper outlines a unified framework to estimate the credibility of published research by examining four fundamental falsifiability-related dimensions: (1) method/data transparency, (2) analytic reproducibility, (3) analytic robustness, and (4) effect replicability. A standardized workflow is proposed to quantify the degree to which a finding has survived scrutiny along these four credibility facets. The framework is demonstrated by applying it to published replications in the psychology literature. A web platform implementation of the framework is outlined, and we conclude by encouraging the community of researchers to contribute to the development and crowdsourcing of the platform.
\end{abstract}

Keywords: research credibility, transparency, open science, analytic reproducibility, analytic robustness, replicability 


\section{A Unified Framework to Quantify the Credibility of Scientific Findings}

Every year, societies spend billions of dollars to fund scientific research aimed at deepening our understanding of the natural and social world. It is expected that some of the insights revealed by that research will lead to applications to address pressing social, medical, and other problems. Published research, however, can only be useful for theory or applications if it is credible. In science, a credible finding or hypothesis is one that has repeatedly survived high-quality, risky attempts at proving it wrong (Lakatos, 1970 Popper, 1959). The more such falsification attempts a finding survives, and the riskier those attempts are, the more credible a finding can be considered.

The currently dominant strategy to assess the credibility of an effect involves metaanalyzing all known studies on a topic (e.g., Cooper, Hedges, \& Valentine, 2009). Such state-of-the-art meta-analytic approaches, however, cannot determine the true credibility of an effect because they do not account for the extent to which each included study has survived risky falsification attempts. For instance, the transparency, analytic credibility, and methodological similarity of meta-analyzed studies are not accounted for (even the standard methods used in Cochrane Reviews of medical research suffer from these limitations; Higgins, Lasserson, Chandler, Tovey, and Churchill, 2018). A credible finding must survive scrutiny along four fundamental kinds of falsifiability-related dimensions: (1) method/data transparency (i.e., availability of study design details, analytic choices, and underlying data), (2) analytic reproducibility (i.e., ability of reported results to be reproduced by repeating the same data processing and statistical analyses on the original data), (3) analytic robustness (i.e., a reported result's being robust to different data processing and data analytic decisions), and (4) effect replicability (i.e., ability to consistently observe an effect of similar magnitude as originally reported in new samples using similar methodologies and conditions as the original study; see Appendix A for more details regarding terminology). If a finding withstands scrutiny along these four dimensions, such that independent researchers fail to identify fatal design flaws, data processing/statistical errors or fragilities, and/or replicability issues, then an effect can be (temporarily) retained as not-yet-falsified and hence treated as credible. The more intense the scrutiny along these four dimensions a finding survives (representing riskier falsification attempts), the more one can be justified in treating it as credible (Popper, 1959).1

\footnotetext{
${ }^{1} \mathrm{~A}$ finding reported with lower levels of method/data transparency due to ethical constraints (e.g., participant privacy and/or confidentiality issues) should nonetheless be considered less credible, all else being equal, compared to a finding reported more transparently. However, such a finding could nonetheless be considered credible if it can be consistently replicated in new samples by independent researchers.
}

Curate Science Version 5.3.0

Corresponding Author: Etienne P. LeBel, Western University, London, Ontario, Canada N6A 3K7, etienne.lebel@gmail.com

M.E. is supported by the Digital Society research program funded by the Ministry of Culture and Science of North Rhine-Westphalia, Germany.

Acknowledgments. We would like to thank E-J Wagenmakers, Daniel Simons, Rogier Kievit, Rolf Zwaan, Alexander Aarts, Touko Kuusi, and Alex Holcombe for valuable feedback on earlier versions of this manuscript. 
Accordingly, to determine credibility, one must assess the degree to which a finding is transparent, reproducible, robust, and replicable. Quantifying these falsifiability-related credibility properties, however, requires a systematic approach because each property is interrelated. Information about one property may influence judgments about the other properties.

Currently, some initiatives do archive information about a study's analytic reproducibility, analytic robustness, and new sample replications (e.g., ReplicationWiki for economics; Harvard Dataverse for political science; PsychFileDrawer.org for psychology; Replication Page for experimental philosophy). Each of these, however, is limited by a lack of standardization, preventing precise estimation of reproducibility, robustness, and replicability across studies and research fields. Concerning reproducibility/robustness archives, no standardized workflow is used to guide researchers on which reproducibility and robustness analyses to conduct and no standardized scoring procedure is used to quantify the degree of reproducibility and robustness observed. Regarding replication archives, the degree of transparency and methodological similarity of replications are not assessed, which preclude the estimation of replicability within and across operationalizations of an effect. Finally, none of these platforms simultaneously consider all such dimensions.

To overcome these limitations, the following paper outlines a single, coherent framework to gauge the credibility of published findings. Uniquely guided by sophisticated falsificationist principles (Lakatos, 1970; Popper, 1959), we propose a standardized workflow to quantify a finding's degree of transparency, reproducibility, robustness, and replicability, and outline a web implementation of the framework currently in development.

\section{Curation Framework}

We propose a unified curation framework to systematically evaluate the credibility of empirical research by quantifying its transparency, reproducibility, robustness, and replicability. Currently, no unified framework exists to assess the degree to which a finding has survived scrutiny along these four dimensions. This is crucial to comprehensively minimize all forms of publication and researcher biases. Further, these dimensions are inherently interrelated and thus should generally be assessed in a particular order 2 Knowledge about one aspect is either necessary for or influences evaluations of the other aspects (e.g., insufficient transparency may prevent the estimation of reproducibility and replicability; lack of robustness may call into doubt the value of executing a replication when expensive designs and/or populations are required). Indeed, it is the only framework that evaluates the transparency, reproducibility, robustness, and replicability of a finding within a harmonized system logically ordered to maximize research efficiency:

1. Transparency: The proposed framework curates compliance to the "basic 4" (LeBel et al., 2013) and more comprehensive reporting standards (e.g., CONSORT, STROBE) for

\footnotetext{
${ }^{2}$ Exceptions may sometimes apply depending on the nature of the study. For example, for inexpensive and easy-to-implement cognitive psychology studies, it may make more sense to evaluate replicability without first gauging analytic reproducibility (though even in this scenario, a study's methodological details should first be thoroughly scrutinized, which requires sufficient method transparency).
} 
any article published in any journal. It also curates and links open practice badges (open materials, open data, and preregistration) to their respective publicly accessible content for any article published in any journal (even those not yet offering badges).

2. Analytic reproducibility: The proposed framework uses a standardized workflow to allow independent analytic reproducibility of a study's primary substantive finding (i.e., primary outcome or set of outcomes), including a scoring procedure to quantify the degree of analytic reproducibility observed.

3. Analytic robustness: The proposed framework employs a standardized workflow to allow independent analytic robustness investigations of a study's primary substantive finding, including a scoring procedure to quantify the degree of analytic robustness observed.

4. Effect replicability: The proposed framework uses a falsifiability-informed approach to organize and evaluate replications of a target effect within and across method and population generalizations of an effect to uniquely address the problems of publication and researcher bias. It curates key replication study characteristics including replication methodological similarity, design differences and deviations, evidence of the plausibility of auxiliary hypotheses (e.g., integrity of instruments), and investigator independence. It uses a novel meta-analytic and individual-study statistical approach to evaluate replication results in a nuanced manner.

\section{Curation of Transparency}

The first, and most fundamental, credibility facet to consider is the degree to which a study's methodological details and data are transparently reported. Without the availability of sufficient methodological details concerning how a study was conducted, it is impossible to comprehensively identify study flaws or data errors and to conduct independent replications. Consequently, the substantive hypothesis tested from a study reported without sufficient transparency is not falsifiable, meaning it is nearly impossible to prove its hypothesis wrong if it is in fact false (Feynmann, 1974). In contrast, a high level of transparency affords relatively high degrees of falsifiability (Popper, 1959), increasing the likelihood that a false hypothesis will be proven wrong.

Four different aspects of transparency should be considered for original and replication studies (in descending order of transparency fundamentality): Compliance to reporting standards for the study design used, open/public materials, preregistration information, and open/public data.

Compliance to reporting standards. Reporting standards are crucial because they specify the precise methodological details of a study that need to be reported for the specific kind of study design employed. With such information transparently reported, researchers are then in a position to identify flaws and confirm that rigorous methodology was indeed used. If such information is not reported, it is impossible to evaluate the rigor of a study ${ }^{3}$

\footnotetext{
${ }^{3}$ One should not however conflate mere compliance with a reporting standard with high levels of methodological rigor.
} 
Prior to 2011, psychology journals did not mandate compliance to official reporting guidelines (though some researchers were advocating that this be done, e.g., Kashy, Donnellan, Ackerman, and Russell, 2009). Demonstrating how easy it is to provide "evidence" for a false conclusion with then-current reporting standards by (un)intentionally exploiting design/analytic flexibility due to researcher biases, Simmons, Nelson, and Simonsohn (2011) proposed a disclosure-based solution whereby authors are required to disclose five basic methodological details about how a study was conducted.

Inspired by Simmons, Nelson, and Simonsohn's (2012) 21-word solution, LeBel et al. (2013) then developed and popularized a "basic 4" reporting standard through their grassroots initiative PsychDisclosure.org (i.e., excluded observations, all tested experimental conditions, all assessed outcome measures, and sample size determination rule). This initiative involved inviting 630 authors of recently published articles to disclose four methodological details not required to be reported but which are crucial for accurate interpretation of published findings. About $50 \%$ of contacted authors voluntarily disclosed such information, and the success of the initiative led psychology's flagship empirical journal Psychological Science (Eich, 2014), and eventually several other journals, to require disclosure of these four methodological details at article submission (LeBel \& John, 2017).

Despite representing an improvement over previous reporting standards in psychology, the "basic 4" reporting standard still falls short of standards that have existed in the medical literature since the 1990s (Begg et al., 1996, e.g., the CONSORT reporting guideline, which specifies 25 methodological details that any randomized controlled trial - or any experimental study - should report). As a starting point, our curation framework proposes that one should at a minimum verify that a study reports methodological details for the "basic 4" categories. In the future, we propose that researchers should curate compliance of their own and others' studies according to more thorough official reporting guidelines.

Open/public materials. "Open materials" is the practice of making accessible in a public repository all experimental materials and procedures required to conduct a fair replication (Kidwell et al., 2016). This practice increases falsifiability of a tested hypothesis by substantially facilitating direct replications by independent researchers. It also increases falsifiability by allowing more thorough scrutiny of study materials and procedures, hence increasing the likelihood that flaws and other methodological shortcomings can be identified.

Preregistration information. Preregistration of study design and analytic plans is crucial with respect to transparency given that it minimizes design and analytic flexibility that can be (un)intentionally exploited (Nosek, Ebersole, DeHaven, \& Mellor, 2018). Preregistration, whether done independently or through a registered report format (Chambers, 2013), allows for more accurate adjustments for multiple analyses, and for clearer distinctions between confirmatory versus exploratory analyses (assuming the preregistration was sufficiently detailed and actually followed). Enhanced transparency in each of these respects also increases falsifiability.

Open/public data. "Open data," like "open materials," is the practice of making accessible at a public repository the raw (or transformed) original data of an article's empirical studies. Such practice increases falsifiability because it allows independent researchers to scrutinize the integrity of the data (e.g., confirming the correct number of participants 
and variables), increasing the likelihood of detecting data errors and internal data inconsistencies. If no serious errors are detected, then a researcher can be more confident regarding the reported results. Higher levels of data transparency in the form of raw compared to transformed data allows for even higher levels of falsifiability.

The proposed framework curates, organizes, and links the open practice badges of open materials/data and preregistration to their respective content at the chosen public repository. This is done at the study-level nested within any article, including those published at journals that do not yet award open practice badges. Curating such information substantially increases the ease of finding the information, and, hence, increases falsifiability by making it easier for other researchers to detect any study design or data errors. Indeed, the full value of increased transparency can only be achieved if such information is actually available and easy to access.

\section{Curation of Analytic Reproducibility and Robustness}

Our curation framework proposes a standardized workflow to gauge the analytic reproducibility and analytic robustness of published findings, including scoring procedures to quantify the degree to which a study's primary reported findings are analytically reproducible and analytically robust.

Analytic reproducibility workflow. The proposed workflow specifies a standardized approach to guide independent researchers in verifying analytic reproducibility, i.e., repeating the same data processing choices and statistical analyses on the original (raw or transformed) data for a study's primary outcome (or set of outcomes) that constitutes the "primary substantive" finding. Following Hardwicke et al. (2018), the primary substantive finding is defined as the one which is emphasized in an article's abstract, figure, or table. The proposed scoring procedure to quantify the degree of analytic reproducibility is the percentage of reproduced outcome effect sizes (ESs) that are consistent with the corresponding originally reported outcomes within a 10\% margin of error (Hardwicke et al., 2018; see Appendix B for more details).

If an independent researcher successfully confirms the analytic reproducibility of a study's reported primary outcome(s), such that no serious discrepancies (greater than a $10 \%$ margin of error) between reproduced and originally reported are detected, then a researcher can be more confident in a study's reported results. Under these conditions, it is then justifiable to investigate the analytic robustness of the reported results. But if results cannot even be reproduced, due to insufficient description of data processing/statistical choices and/or discrepant results, then confidence in the reported results should be reduced. In such a situation, it is unclear whether the expenditure of time or resources to evaluate the analytic robustness of a study's reported results is justified.

Analytic robustness workflow. The proposed workflow and scoring procedure to evaluate analytic robustness roughly parallels the analytic reproducibility workflow. The workflow is informed by Steegen, Tuerlinckx, Gelman, and Vanpaemel's (2016) multiverse analysis approach, which estimates the extent to which a study's conclusions are robust to 
reasonable alternative data processing choices that could also have been conducted by examining the frequency of p-values for all combinations (or "multiverse") of all such alternative data processing choices. Analytic robustness also is informed by Simonsohn, Simmons, and Nelson's (2015) specification curve analysis, which examines a study's primary effect estimates of all reasonable combinations of alternative data processing and statistical analyses (what they call "specifications") that could have been conducted, and estimating the extent to which the set of such estimates is inconsistent with the null hypothesis.

The standardized workflow involves examining ES estimates, with corresponding confidence intervals, of all reasonable combinations of alternative data processing choices and statistical analytic models for a study's primary outcome (or set of outcomes) that constitutes the "primary substantive" finding (as defined to estimate reproducibility). The proposed scoring procedure to quantify the degree of analytic robustness of a reported result is the percentage of such multiverse ES estimates that are consistent with the point estimate of the originally reported ES estimate. When reported results appear to be analytically robust (e.g., $>80 \%$ of the multiverse ES estimates are consistent with their corresponding original ES point estimate), it is then justifiable to consider evaluating the replicability of the tested target substantive hypothesis (assuming the study's methodology has been sufficiently transparently reported as described in section 1). In contrast, if a reported result is not analytically robust (i.e., is fragile to, or highly contingent upon, different data processing choices and analytic models), then evaluating effect replicability may not be justified depending on the resource costs and/or feasibility of conducting independent replications of the target study (e.g., it may not be justified to conduct a replication of a study involving a longitudinal study or a difficult-to-recruit population).

\section{Curation of Effect Replicability}

Our approach estimates effect replicability by specifying (1) a flexible workflow to organize replications within and across distinct operationalizations of a target effect (which also allows gauging the generalizability of an effect), (2) curation of key replication study characteristics including methodological similarity relative to an original study using a principled replication taxonomy, replication design differences/deviations, evidence of auxiliary hypotheses plausibility, and investigator independence, and (3) a meta-analytic and individual-study level statistical approach to evaluate replication results in a nuanced manner. Such falsifiability-informed and stringent approach minimizes all forms of publication and researcher biases, as elaborated upon below.

Flexible workflow to organize replications. The proposed workflow assesses the replicability of an effect by organizing replications within and across distinct operationalizations (or "generalizations") of an effect. Replicability is gauged across sufficiently methodologically similar replications within distinct operationalizations of an effect (see Appendix C for a diagram of this organizational structure). Generalizations can either be method generalizations (generalizations of an effect to different general methodologies for the IV and/or DV/paradigm) or population generalizations (generalizations of an effect to a different population than was used in an original study). The generalizability of an effect 
can then be evaluated by examining the degree to which an effect is replicable across distinct generalizations of an effect.

Curation of key replication study characteristics. The key replication study characteristics curated include (1) methodological similarity relative to an original study using a principled replication taxonomy, (2) replication design differences/deviations, (3) evidence of the plausibility of auxiliary hypotheses (e.g., integrity of instruments), and (4) investigator independence (see Appendix D for more details regarding these key replication study characteristics). Before turning to these, it should be mentioned that the transparency (compliance to reporting standards, open materials, preregistration information $]^{4}$ and open data) and state of analytic reproducibility and robustness (see above) should also be examined and considered for replication studies, given that such study characteristics are crucial to verify for all studies.

Replication methodological similarity. To be eligible for inclusion in a replication evidence collection, a replication study must employ a sufficiently similar methodology (Earp, in press). To guide the classification of replications based on methodological similarity to an original study, we use the replication taxonomy depicted in Figure 1, introduced by LeBel, Berger, Campbell, and Loving (2017), which is a simplified version of earlier taxonomies (Hendrick, 1991; Schmidt, 2009). According to this taxonomy, different types of increasingly dissimilar replications exist between the highly similar and highly dissimilar poles, each serving different purposes (for more details and examples of each replication type, see Appendix C).$^{5}$

Each type of replication serves different epistemological purposes (Zwaan, Etz, Lucas, \& Donnellan, 2017). We consider only direct replications ("Exact", "Very Close", and "Close" replications) as sufficiently similar to an original study. The reason for this is that only the results of such types of replications can in principle - across several replication attempts - falsify a hypothesis (assuming sound auxiliary hypotheses) and consequently cast doubt upon the credibility of an effect (Earp \& Trafimow, 2015; Meehl, 1967, 1978). By contrast, the major - and intentionally introduced - methodological differences of "Far" and "Very Far" replications (i.e., generalizations) can never cast doubt on an originally reported effect (Doyen, Klein, Simons, \& Cleeremans, 2014). This is because unsupportive evidence from such studies is ambiguous: It could be due to the falsity of the original hypothesis or to one or more of the changes in methodology in the replication attempt (Pashler \& Harris, 2012). Hence, such studies can only speak to the generalizability of a presumably-replicable effect.

In summary, only direct replications with sufficiently similar methodology, which naturally constrain design/analytic flexibility, can provide the sort of strict falsification attempt

\footnotetext{
${ }^{4}$ Given that replication studies within our framework need to be sufficiently methodological similar relative to an original study, they can be construed as tacitly "preregistered." However, formally preregistering design and analytic plans of replication studies can nonetheless further constrain more minor forms of design/anaytic flexibility.

${ }^{5}$ Unless preceded by a modifier (e.g., "far"), we use the term "replication" to refer to "direct replication" and "generalization" to refer to "conceptual replication."
} 


\begin{tabular}{|c|c|c|c|c|c|}
\hline \multirow[b]{3}{*}{ Design facet } & \multicolumn{4}{|c|}{ Replication continuum } & \multirow{2}{*}{$\begin{array}{l}\text { Highly dissim } \\
\text { | replication }\end{array}$} \\
\hline & \multicolumn{3}{|c|}{ Direct replication } & Conceptual replication & \\
\hline & $\begin{array}{l}\text { Exact } \\
\text { replication } \\
\text { (All facets under } \\
\text { researcher control } \\
\text { are the same) }\end{array}$ & $\begin{array}{c}\text { Very close } \\
\text { replication } \\
\text { (Procedures or } \\
\text { physical setting are } \\
\text { different) }\end{array}$ & $\begin{array}{l}\text { Close } \\
\text { replication } \\
\text { (IV or DV stimuli } \\
\text { are different) }\end{array}$ & $\begin{array}{c}\text { Far replication } \\
\text { (IV or DV } \\
\text { operationalizations } \\
\text { or population are } \\
\text { different) }\end{array}$ & $\begin{array}{c}\text { Very Far } \\
\text { replication } \\
\text { (IV or DV } \\
\text { constructs are } \\
\text { different) }\end{array}$ \\
\hline Effect/hypothesis & same & same & same & same & same \\
\hline IV construct & same & same & same & same & different \\
\hline DV construct & same & same & same & same & different \\
\hline IV operationalization & same & same & same & different & \\
\hline DV operationalization & same & same & same & different & \\
\hline Population (e.g., age) & same & same & same & different & \\
\hline IV stimuli & same & same & different & & \\
\hline DV stimuli & same & same & different & & \\
\hline Procedural details & same & different & & & \\
\hline Physical setting & same & different & & & \\
\hline Contextual variables & different & & & & \\
\hline : & $\vdots$ & & & & \\
\hline
\end{tabular}

Figure 1. Replication taxonomy to guide the classification of the methodological similarity of a replication study relative to an original study. "Same" ("different") indicates the design facet in question is the same (different) compared to an original study. IV = independent variable, DV = dependent variable. Procedural details involve minor experimental particulars (e.g., task instructions, font, font size, etc.). "Contextual variables" reflect design facets.

whose survival justifies increased confidence in a target hypothesis. Such an approach contrasts sharply with the traditional meta-analytic approach, which cannot yield trustworthy meta-analytic conclusions because it combines incomparable studies of unknown methodological similarity and unknown levels of transparency, reproducibility, and robustness (for more details about inadequacies of traditional meta-analyses, see Appendix D).

Replication design differences/deviations. Design differences and deviations involve any study design characteristics, within or beyond the researcher's control, that were different compared to an original study. These are important to consider for accurate interpretation of a replication result. For positive replication evidence, knowing about such design differences shows that an effect is robust across these differences. For negative replication evidence, such differences provide initial clues regarding potential boundary conditions of an effect.

Auxiliary hypotheses plausibility evidence. To test a substantive hypothesis, several auxiliary hypotheses must be assumed to hold true (e.g., that the measurement instruments operated correctly; that participants understood the instruction and paid sufficient attention; Meehl, 1967). When interpreting study results, it is important to consider different pieces of evidence that help gauge how plausible it was that such auxiliary hypotheses were sound (LeBel \& Peters, 2011)). Consequently, such information (also known as "positive controls"; Moery and Calin-Jageman, 2016) is a key study characteristics that 
is curated and should be considered when interpreting replication results. This is particularly important in situations where a replication study reports a null finding, to rule out more mundane explanations for not having detected a target effect. For example, evidence of a successful manipulation check or detecting a known replicable effect (e.g., a semantic priming effect) helps rule out that a fatal experimenter error or data processing error caused the observed null finding.

Investigator independence. This refers to basic information about the degree of independence between the replication investigators compared to the researchers who conducted the original study. Investigator independence is important to protect against confirmation and other biases (Earp \& Trafimow, 2015, Rosenthal, 1991).

Curating these four key replication study characteristics helps researchers evaluate the replicability of an effect in a nuanced fashion by allowing them to weight replications according to the aforementioned dimensions (e.g., give more weight to more transparently reported and/or analytically reproducible replications; give less weight to replications conducted by non-independent researchers). Such information also allows researchers to quantitatively meta-analyze different subsets of replications that vary on these study characteristics.

Principled statistical approach to evaluating replication evidence. The next step is then to use a principled approach to statistically evaluate replication evidence at both (1) the individual replication study level (when only one or a few replications are available) and (2) at the meta-analytic level (when several replications are available) by synthesizing evidence across replication studies nested within distinct generalizations of an effect (the original study effect is not included in the meta-analytic estimate). Whether statistically evaluating replication evidence from an individual study or meta-analytically, we propose a statistical approach that is more nuanced than what is currently standard practice in the field and that also uses clearer language to describe replication results (see also LeBel, Vanpaemel, Cheung, and Campbell, 2018). This approach considers three distinct statistical aspects: (1) whether a signal was detected, (2) the consistency of the replication effect size (ES) relative to the original study ES, and (3) the precision of the replication ES estimate. For example, in replication scenario \#1 (see Figure 2), we would say that a signal was detected (given that the replication ES confidence interval excludes zero) and that it is consistent with the original study (given that the replication ES confidence interval includes the original ES point estimate). This represents the most favorable replication outcome in terms of surviving a severe falsifying test. In contrast, in replication scenarios $\# 2, \# 3$, and \#4, a signal was detected but the replication ES is inconsistent with the original ES point estimate, a less favorable replication outcome suggesting that boundary conditions of the target effect may not yet be well understood. Finally, replication scenarios \#5 and \#6 reflect even less favorable replication evidence given that no signal was detected, with scenario \#6 representing the least favorable outcome given that the replication ES estimate is inconsistent with the original ES point estimate.

Following this terminology, an effect can be considered "replicable" when replications consistently detect a signal each of which is consistent (i.e., of similar magnitude) with the ES point estimate from the original study (replication scenario \#1). Meta-analytically, 


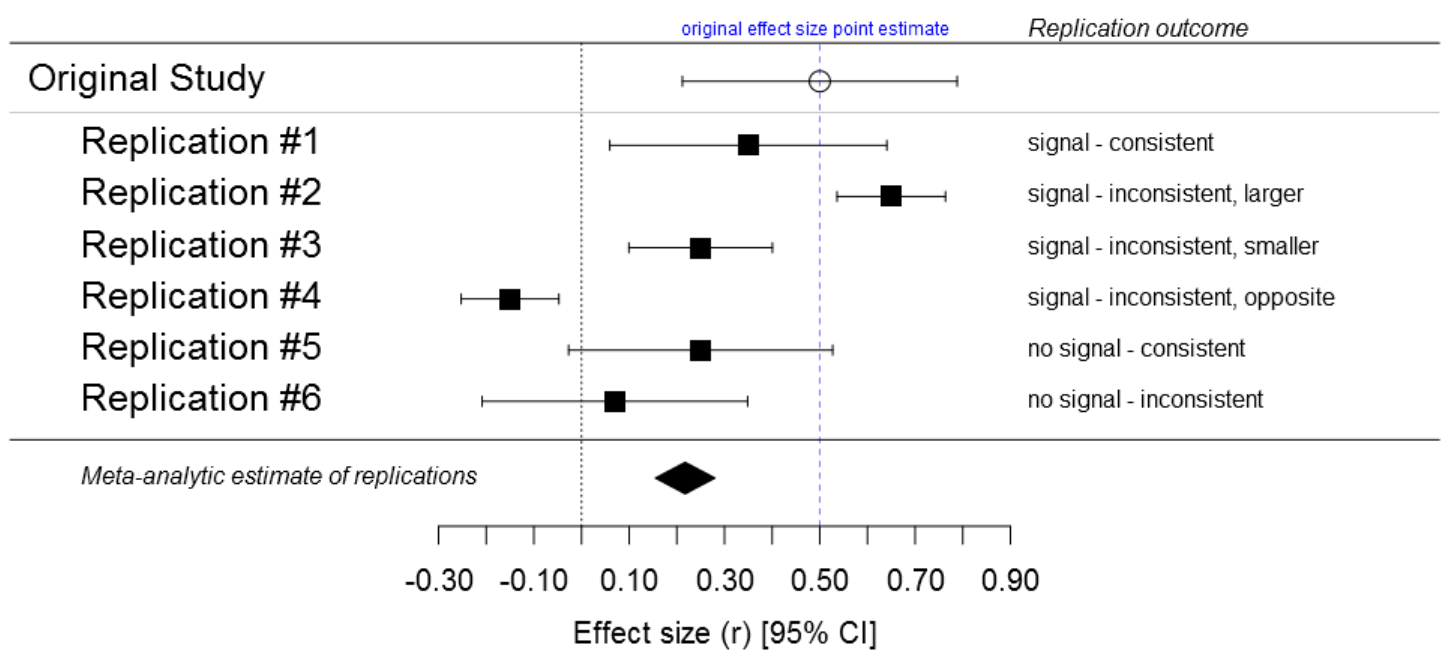

Figure 2. Six distinct hypothetical outcomes of a replication study based on considering three statistical aspects of a replication result: (1) whether a signal was detected, (2) the consistency of the replication effect size (ES) relative to the original study, and (3) the precision of the replication ES estimate (from Figure 1 of LeBel, Vanpaemel, Cheung, and Campbell, 2018; CC-By Attribution 4.0 International).

when several replications are available for a specific operationalization of an original effect, an effect is "replicable" when the meta-analytic ES estimate excludes zero and is consistent with the original ES point estimate.

\section{Curate Science Web Platform}

The proposed unified curation framework guides the design and implementation of a crowdsourced searchable web platform (CurateScience.org) that allows the community of researchers to curate and evaluate the transparency, reproducibility, robustness, and replicability of each other's findings in an incremental, ongoing basis. A non-static web platform is crucial because scientific evidence is dynamic and constantly evolving: new evidence can always count against, or be consistent with, a previously accepted hypothesis. In the digital era, it no longer makes sense to continue publishing literature reviews of evidence as static documents that become out-of-date shortly after they are submitted to a journal for peer-review (as in traditional meta-analyses). The crowdsourced, incremental platform is decentralized and thus the contributed evidence can (1) be as inclusive as possible, (2) originate from maximally diverse researchers in terms of intellectual and theoretical viewpoints, and (3) be up-to-date.

The platform will feature two main screens: one for original studies and another for replication studies. Users will be able to search for (and filter) studies based on study 
characteristics related to transparency, reproducibility, robustness, and replicability. This will allow researchers to search for articles that comply with minimum levels of different kinds of transparency (e.g., only show articles that report preregistered studies with open study materials; only show articles with publicly available data and reproducible code files).

Concerning transparency, researchers will be able to indicate that their studies (already) comply with a specific reporting standard (e.g., "basic 4") and/or retroactively disclose missing/unreported information so that their studies comply with a chosen standard. A standardized labeling system will be used to display whether a study complies with a reporting standard, and, if so, which one (and also display additionally disclosed information in the case of retroactive disclosure statements; see Figure 3). This is crucial given that only a minority of journals require compliance to such standards and those that do, do not use a standardized labeling system 6 Researchers will also be able to "earn" open practice badges for studies published in journals that do not yet award these badges, by allowing the hyperlinking of the relevant open practice badge icons to the URLs of the publicly available resources (i.e., open study materials, preregistered study protocols, and open data/reproducible code files).

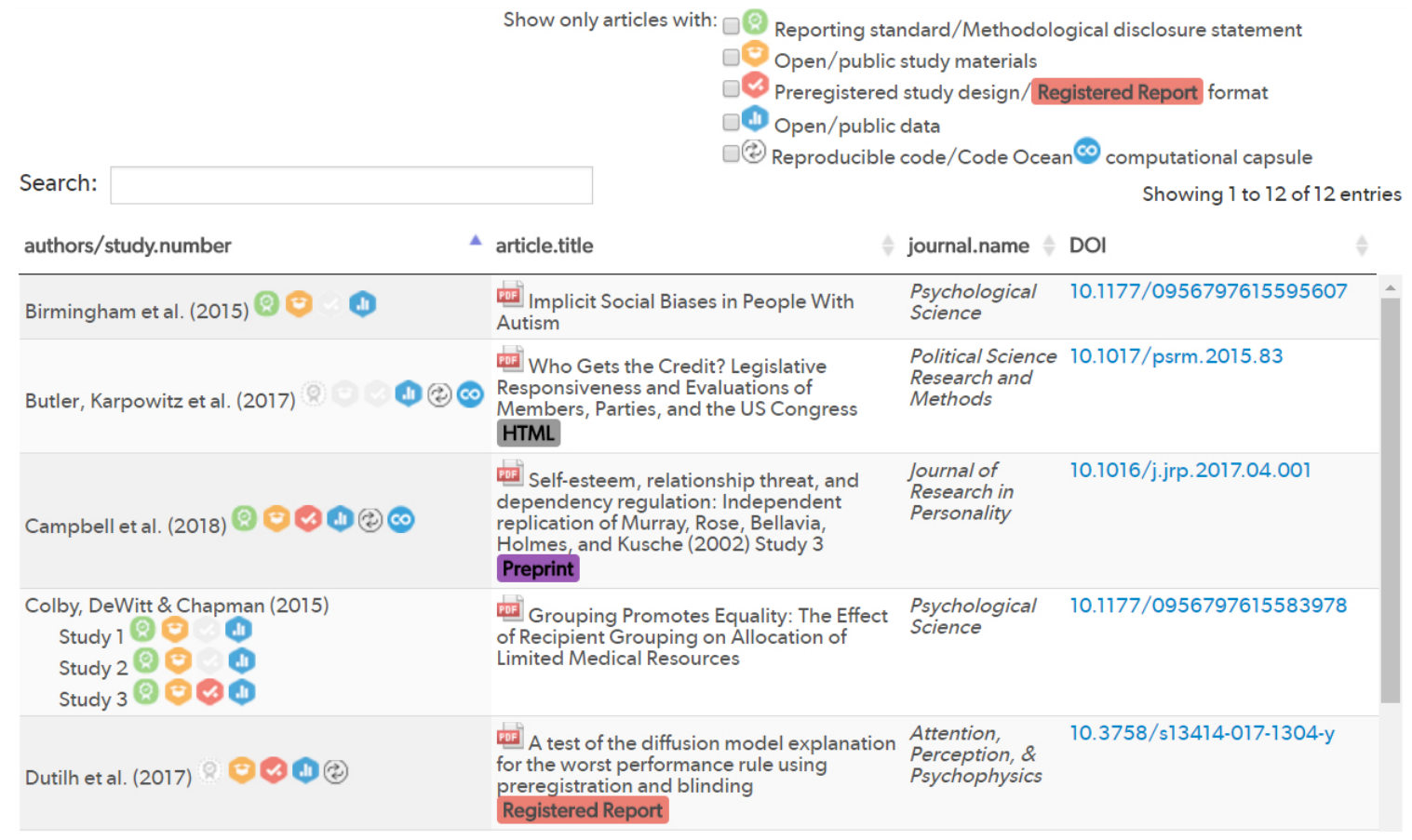

Figure 3. Search for transparently reported studies prototype (interactive prototype available at http://curatescience.org/\#search-for-transparent-studies-section). Researchers will be able to add and curate their own transparently reported articles by linking the relevant open practice badge icons to the URLs of the publicly available study components.

\footnotetext{
${ }^{6}$ The platform will also allow researchers to leave comments regarding study flaws and other identified issues (and will also allow them to add hyperlinks to other published commentaries/critiques about the study; e.g., from PubPeer.com or blog posts).
} 
Concerning reproducibility and robustness, users will be able to upload (and get credit for) analytic reproducibility and robustness verifications of a study's primary substantive finding. In the falsifiability spirit, it is crucial that such verifications are themselves easily scrutinizable so that they can be verified by independent researchers (see Appendix D for UI prototype showing how such verifications will be displayed in search results).

Finally, users will be able to add missing replications to a pre-existing evidence collection of a target effect, and be able to create new evidence collections of replications of effects not yet available in the database. For available replications, researchers will be able to meta-analyze replication evidence of selected replications (within one's web browser) based on curated key replication study characteristics (e.g., methodological similarity, design differences/deviations, preregistration status, etc.; see Appendix D for UI prototype).

The success of the platform will hinge on the active involvement of researchers to engage with the website and contribute content (e.g., add missing replications, curate study information, perform reproducibility analyses, etc.). To incentivize the contribution and curation of content, and also to maximize the quality of the contributed content, the platform will include key features guided by social accountability and reward principles. 7 For example, all of a user's contributions will be prominently displayed on their public profile page and recent contributions will also be conspicuously displayed on the home page (displaying the contributor's name, which can be clicked to see that researcher's profile page). A "low barrier to entry" incremental approach will be used to maximize the number and frequency of contributions, achieved by having as many "optional" fields as possible, so that the curation of information can be continued later by other users and/or editors. To maximize the quality of the contributed content, the platform will track the user name and date of all added and updated information and will also feature light-touch editorial review for certain categories of information (e.g., when a new replication is added to an existing evidence collection, the information will appear as "unverified" until another user or editor reviews it).

\section{Example: Infidelity Distress Effect}

To demonstrate our proposed framework, we apply it to replications of the infidelity distress effect (Buss et al., 1999, Study 2; see Appendix E for two additional examples). According to this effect, men, compared to women, are on average more likely to report being more distressed by a (hypothetical) sexual versus emotional infidelity committed by their partner (forced choice). The original instantiation of the effect was documented in a sample of young individuals (i.e., undergraduate students; Buss et al., 1999, Study 2). In a population generalization, the effect was generalized to an older community sample of individuals (i.e., mean age $=67.1$ years; Shackelford et al., 2004).

\footnotetext{
${ }^{7}$ To further encourage contributions, and as is standard for crowdsourced platforms, during initial phases, we will pay (PhD-level) curators to contribute content and consequently seed the database to sufficient levels to convince other users that the platform is wide-ranging enough to be worth contributing to. As of May 2018, the website features $N=1,058$ partially-curated replications of 168 effects from the cognitive and social psychology literature.
} 


\section{Transparency}

The methodological details of the original studies were not disclosed according to the "basic 4" or more comprehensive reporting standards, though it did comply with the reporting standards at the time. The original studies do not qualify to earn open practice badges, nor were they preregistered given that the research was conducted before the advent of such practice.

\section{Analytic reproducibility/robustness}

Given that the data for Buss et al.'s (1999) and Shackelford et al.'s (2004) original studies are not publicly available, analytic reproducibility and robustness verifications are not possible.

\section{Effect Replicability}

Regarding study characteristics of the individual replication studies, each replication complied with the "basic 4" reporting standard, has open materials, open data, and was also preregistered. The open data allows independent verifications of analytic reproducibility of the reported results. Indeed, the first author attempted this and was successfully able to reproduce the reported primary outcome effect sizes (within a $10 \%$ margin of error) for all five of IJzerman et al.'s (2014) replications. The preregistered nature of the replications also helps rule out the possibility that more minor forms of analytic and design flexibility biased the results (assuming that the preregistration was sufficiently detailed and that the study procedures reported in the paper followed the preregistered protocol). Though no evidence of positive controls were reported, the open data nature of the studies means that auxiliary hypotheses plausibility evidence can be inferred from examining internal consistency estimates of individual difference measures assessed. For example, the sociosexual orientation inventory (SOI) measure exhibited high internal consistency estimates $(\alpha=.87$, $\alpha=.85, \alpha=.80$, and $\alpha=.86$ across IJzerman et al.'s (2014) Studies 1 through 4, respectively), suggesting it is plausible that auxiliary hypotheses were sound. In terms of design differences, all replication studies were conducted in Dutch (instead of English), IJzerman et al.'s (2014) Study 4 was conducted online (instead of in the lab), and the infidelity distress measure involved eight dilemmas (provided by original authors) instead of six. All replications involved independent investigators.

Concerning replicability and generalizability evidence, as can be seen in Figure 4, for the original instantiation of the effect among young individuals, meta-analytic replication evidence reveals an infidelity distress effect of $d=.57+/-.39$ (not including the original study ES estimate).

Thus a signal was detected; however, the meta-analytic replication ES estimate confidence interval excludes the original study's ES point estimate of $d=1.30$, hence is considered inconsistent with the original study (scenario \#3). This suggests that the original study may have overestimated the effect magnitude and/or that boundary conditions for 

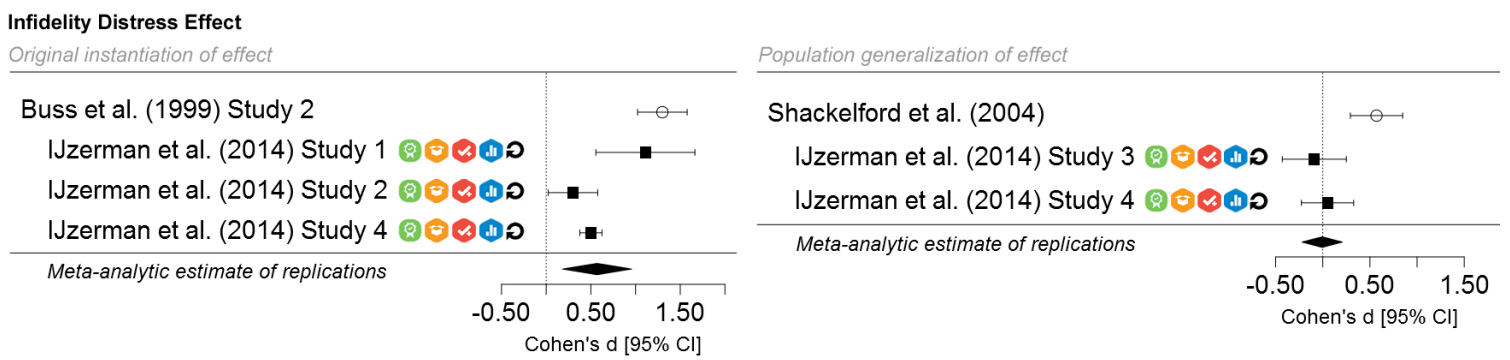

Figure 4. Replications of original instantiation and a population generalization of the infidelity distress effect (Buss et al., 1999, Study 2; Shackelford et al., 2004). As indicated by the icons, each replication complied with the "basic 4" reporting standard, has open study materials, open data, was preregistered, and was confirmed to be analytically reproducible.

the effect are still not well understood. For the population generalization, no signal was detected (meta-analytic replication ES estimate of $d=-.01+/-.21$, which is inconsistent with the original study's ES point estimate of $d=.57$; scenario \#6), suggesting that the effect may not generalize to older individuals.

\section{Conclusion}

The current article proposes a unified framework to systematically quantify the method/data transparency, analytic reproducibility, analytic robustness, and effect replicability of published scientific findings. The framework is unique compared to extant approaches in several ways. It is the only framework that integrates "deep-level" curation of transparency, reproducibility, robustness, and replicability of empirical research in a harmonized, flexible system that is logically ordered to maximize research efficiency. It also uniquely curates, at the study-level, the transparency of published findings (i.e., compliance to reporting standards, open materials, preregistration information, open data), and advances standardized workflows and scoring procedures to estimate the degree of reproducibility and robustness of reported results. It also proposes a novel system to organize and evaluate the replicability of effects by curating key characteristics of replication studies and statistically evaluating replication results in a nuanced manner at the meta-analytic and individual-study level.

In conclusion, it is important to mention what the unified framework, or its web implementation, is not. It is not a debunking platform aimed at cherry-picking unfavorable replicability evidence of published findings. It is also not a "final authoritative arbiter" of research quality. In contrast, it is a system to organize scientific information and develop meta-scientific tools to help the community of researchers carefully evaluate research in a nuanced manner. It is also not a private club, but rather an open, decentralized, and transparently accountable public resource available to all researchers who abide by the relevant scientific codes of conduct and civility norms of communication. 
Table 1

Benefits and value created by curating the transparency, reproducibility, robustness, and replicability of empirical research.

\begin{tabular}{ll}
\hline Benefit Category & Benefit/Value \\
\hline Theory building and & - It allows researchers to base beliefs about the credibility of \\
application & effects on empirical evidence rather than authority (e.g., \\
& journal or university prestige). \\
& ready to be extended (particularly useful for graduate \\
& students and early-career researchers). \\
& - It allows researchers to more accurately estimate effect sizes \\
& within a research area, yielding better estimates of sample \\
& sizes needed to achieve sufficient statistical power. \\
& - It allows researchers to identify important findings that \\
& have not yet been replicated and commission the conduct of \\
& such replications (via e.g., StudySwap or the Psychological \\
& Science Accelerator).
\end{tabular}

Meta-scientific

- It yields a rich database of transparently reported studies and replications that can be used for meta-science research to deepen our understanding of the predictors of replicability (e.g., original study p-value, sample size, study design).

- The platform can be used to track the transparency, reproducibility, robustness and replicability of disciplines over time to gauge progress in achieving higher research integrity.

Teaching/pedagogical

- The searchable database can be used to teach about transparency and replication (e.g., showing real world examples of effects exhibiting different levels of replicability; it can also inform teachers about replicable effects that can justifiably be taught).

Practical

- It helps researchers locate publicly-available experimental materials for follow-up research and publicly available data sets/reproducible code files for secondary (re-)analyses from alternative theoretical perspectives.

Social normative

- Making it easier to find more transparently reported research increases the likelihood that ambivalent or unaware researchers will decide to adopt such transparent practices, hence accelerating a cultural shift in the research community where it becomes the social norm to report one's research more transparently.

- By increasing the visibility of replication studies, the platform rewards the contributions of researchers who devote their time replicating the work of others. 
Crowdsourcing the credibility of published research creates value and is expected to lead to several distinct benefits (see Table 1).

We hope that the current article will serve as a call to action for the research community to get involved in using, designing, and contributing to the web platform CurateScience.org. The vision is that of a vibrant community of users and researchers who use and contribute to the platform in a collective bid to digitally organize our published literature. This crowdsourcing of the credibility of empirical research will consequently accelerate our theoretical understanding of the world as well as the development of applied solutions to society's most pressing social and medical problems. 


\section{References}

Begg, C., Cho, M., Eastwood, S., Horton, R., Moher, D., Olkin, I., .. Stroup, D. F. (1996). Improving the quality of reporting of randomized controlled trials. JAMA, 276(8), 637-639. doi:10.1001/jama.1996.03540080059030

Buss, D. M., Shackelford, T. K., Kirkpatrick, L. A., Choe, J. C., Lim, H. K., Hasegawa, M., ... Bennett, K. (1999). Jealousy and the nature of beliefs about infidelity: Tests of competing hypotheses about sex differences in the United States, Korea, and Japan. Personal Relationships, 6(1), 125-150. doi:10.1111/j.1475-6811.1999.tb00215.x

Chambers, C. D. (2013). Registered reports: A new publishing initiative at Cortex. Cortex, 49(3), 609-610. doi:10.1016/j.cortex.2012.12.016

Cooper, H., Hedges, L. V., \& Valentine, J. C. (Eds.). (2009). The handbook of research synthesis and meta-analysis. New York, NY: Russell Sage Foundation.

Doyen, S., Klein, O., Simons, D. J., \& Cleeremans, A. (2014). On the other side of the mirror: Priming in cognitive and social psychology. Social Cognition, 32, 12-32. doi:10.1521/ soco.2014.32.supp.12

Earp, B. D. \& Trafimow, D. (2015). Replication, falsification, and the crisis of confidence in social psychology. Frontiers in Psychology, 6. doi:10.3389/fpsyg.2015.00621

Eich, E. (2014). Business not as usual. Psychological Science, 25(1), 3-6. doi:10.1177/ 0956797613512465

Feynmann, R. P. (1974). Cargo cult science. Engineering \& Science, 37(7), 10-13.

Hardwicke, T. E., Mathur, M. B., MacDonald, K., Nilsonne, G., Banks, G. C., Kidwell, M. C., ... Frank, M. C. (2018). Data availability, reusability, and analytic reproducibility: Evaluating the impact of a mandatory open data policy at the journal Cognition. doi:10.17605/OSF.IO/39CFB

Hendrick, C. (1991). Replication, strict replications, and conceptual replications: Are they important? In J. W. Neuliep (Ed.), Replication research in the social sciences (pp. 4149). Newbury Park, CA: Sage.

Higgins, J., Lasserson, T., Chandler, J., Tovey, D., \& Churchill, R. (2018). Mmetodological expectations of Cochrane intervention reviews (MECIR): Standards for the conduct and reporting of new cochrane intervention reviews, reporting of protocols and the planning, conduct and reporting of updates ( $v$ 1.05). Retrieved from http://community. cochrane.org/mecir-manual

IJzerman, H., Blanken, I., Brandt, M. J., Oerlemans, J. M., Van den Hoogenhof, M. M. W., Franken, S. J. M., \& Oerlemans, M. W. G. (2014). Sex differences in distress from infidelity in early adulthood and in later life. Social Psychology, 45 (3), 202-208. doi:10. 1027/1864-9335/a000185

Kashy, D. A., Donnellan, M. B., Ackerman, R. A., \& Russell, D. W. (2009). Reporting and interpreting research in PSPB: Practices, principles, and pragmatics. Personality and Social Psychology Bulletin, 35(9), 1131-1142. doi $10.1177 / 0146167208331253$

Kidwell, M. C., Lazarević, L. B., Baranski, E., Hardwicke, T. E., Piechowski, S., Falkenberg, L.-S., ... Nosek, B. A. (2016). Badges to acknowledge open practices: A simple, lowcost, effective method for increasing transparency. PLOS Biology, 14(5), e1002456. doi:10.1371/journal.pbio.1002456 
Lakatos, I. (1970). Falsification and the methodology of scientific research programmes. In I. Lakatos \& A. Musgrave (Eds.), Criticism and the growth of knowledge (pp. 91-196). Cambridge: Cambridge University Press. doi:10.1017/CBO9781139171434.009

LeBel, E. P., Berger, D., Campbell, L., \& Loving, T. J. (2017). Falsifiability is not optional. Journal of Personality and Social Psychology, 113(2), 254-261. doi:10.1037/ pspi0000106

LeBel, E. P., Borsboom, D., Giner-Sorolla, R., Hasselman, F., Peters, K. R., Ratliff, K. A., \& Smith, C. T. (2013). PsychDisclosure.org: Grassroots support for reforming reporting standards in psychology. Perspectives on Psychological Science, 8(4), 424-432. doi:10. $1177 / 1745691613491437$

LeBel, E. P. \& John, L. K. (2017). Toward transparent reporting of psychological science. In S. O. Lilienfeld \& I. D. Waldman (Eds.), Psychological science under scrutiny (pp. 7384). Hoboken, NJ: Wiley. doi:10.1002/9781119095910.ch5

LeBel, E. P. \& Peters, K. R. (2011). Fearing the future of empirical psychology: Bem's (2011) evidence of psi as a case study of deficiencies in modal research practice. Review of General Psychology, 15 (4), 371-379. doi $10.1037 / \mathrm{a} 0025172$

LeBel, E. P., Vanpaemel, W., Cheung, I., \& Campbell, L. (2018). A guide to evaluate replications: A comment on Zwaan et al. (2017). Manuscript under review at MetaPsychology. doi:10.17605/OSF.IO/PAXYN

Meehl, P. E. (1967). Theory-testing in psychology and physics: A methodological paradox. Philosophy of Science, 34 (2), 103-115. doi:10.2307/186099

Meehl, P. E. (1978). Theoretical risks and tabular asterisks: Sir Karl, Sir Ronald, and the slow progress of soft psychology. Journal of Consulting and Clinical Psychology, 46(4), 806-834. doi:10.1037/0022-006X.46.4.806

Moery, E. \& Calin-Jageman, R. J. (2016). Direct and conceptual replications of Eskine (2013). Organic food exposure has little to no effect on moral judgments and prosocial behavior. Social Psychological and Personality Science, 7(4), 312-319. doi:10.1177/ 1948550616639649

Nosek, B. A., Ebersole, C. R., DeHaven, A. C., \& Mellor, D. T. (2018). The preregistration revolution. Proceedings of the National Academy of Sciences, 115(11), 2600-2606. doi:10.1073/pnas.1708274114

Pashler, H. \& Harris, C. R. (2012). Is the replicability crisis overblown? Three arguments examined. Perspectives on Psychological Science, 7(6), 531-536. doi 10.1177/ 1745691612463401

Popper, K. R. (1959). The logic of scientific discovery. London, UK: Hutchinson.

Rosenthal, R. (1991). Replication in behavioral research. In J. W. Neuliep (Ed.), Replication research in the social sciences (pp. 1-39). Newbury Park, CA: Sage.

Schmidt, S. (2009). Shall we really do it again? The powerful concept of replication is neglected in the social sciences. Review of General Psychology, 13(2), 90-100. doi:10. $1037 / \mathrm{a} 0015108$

Shackelford, T. K., Voracek, M., Schmitt, D. P., Buss, D. M., Weekes-Shackelford, V. A., \& Michalski, R. L. (2004). Romantic jealousy in early adulthood and in later life. Human Nature, 15(3), 283-300. doi:10.1007/s12110-004-1010-z 
Simmons, J. P., Nelson, L. D., \& Simonsohn, U. (2011). False-positive psychology: Undisclosed flexibility in data collection and analysis allows presenting anything as significant. Psychological Science, 22 (11), 1359-1366. doi:10.1177/0956797611417632

Simmons, J. P., Nelson, L. D., \& Simonsohn, U. (2012). A 21 word solution. SSRN Electronic Journal. doi:10.2139/ssrn.2160588

Simonsohn, U., Simmons, J. P., \& Nelson, L. D. (2015). Specification curve: Descriptive and inferential statistics on all reasonable specifications. SSRN Electronic Journal. doi: $10.2139 /$ ssrn.2694998

Steegen, S., Tuerlinckx, F., Gelman, A., \& Vanpaemel, W. (2016). Increasing transparency through a multiverse analysis. Perspectives on Psychological Science, 11(5), 702-712. doi: $10.1177 / 1745691616658637$

Zwaan, R. A., Etz, A., Lucas, R. E., \& Donnellan, M. B. (2017). Making replication mainstream. Behavioral and Brain Sciences. doi:10.1017/S0140525X17001972 


\section{Author Contributions}

EPL conceived the general idea, drafted the manuscript, created the figures and diagrams, and executed the analytic reproducibility checks and evidence collection metaanalyses. WV provided substantial contributions to conceptual developments. WV, RM, $\mathrm{BDE}$, and ME provided substantial contributions to the writing, writing revisions, critical commentary, and manuscript edits. EPL drafted major revisions of the manuscript, with WV, RM, and BDE contributing critical feedback and manuscript edits. All authors approve the final submitted version of the manuscript. 